\title{
On the Dual Effect in State-Based Scheduling of Networked Control Systems
}

\author{
Chithrupa Ramesh, Henrik Sandberg, Lei Bao and Karl Henrik Johansson
}

\begin{abstract}
In this paper, we show that there is a dual effect with state-based scheduling. In general, this makes the optimal scheduler and controller hard to find. However, by removing past controls from the scheduling criterion, we find that certainty equivalence holds. This condition is related to the classical result of Bar-Shalom and Tse, and it leads to the design of a sub-optimal scheduler with a certainty equivalent controller. Furthermore, we show that a mapping of the statebased scheduler into one which fulfills this condition, and consequently has an optimal certainty equivalent controller, does not result in an equivalent class of design in the sense of Witsenhausen. Computing the estimate remains hard, but can be simplified by introducing a symmetry constraint on the scheduler.
\end{abstract}

\section{INTRODUCTION}

There is a need for innovative scheduling policies in networked control systems. These systems aim to support the regulation of physical plants over wireless networks. The push to wireless is motivated by a need for mobility and reduced cabling and installation costs, among other benefits. But, scheduling over wireless networks is more demanding due to the interference-limited nature of these networks. Also, control applications may share access to the wireless medium with non-regulatory and non-critical applications, which makes the problem challenging. This calls for a two-pronged approach: to limit access to the communication channel from the application, and to ensure that performance guarantees are met by the scheduler. The first approach has driven the design of event-based sampling systems [1], which seek to reduce the traffic in the network by transmitting only when required. This is often achieved by assigning a cost for every channel-use [2], and minimizing the net cost of controlling the plant and communicating over the medium. The design of the optimal scheduler, in this sense, might be enabled by providing the scheduler access to all the information about the plant including the state and its evolution, the applied control signals, the disturbances and the cost criterion. The second approach tries to ensure performance guarantees from the scheduler, which makes the design of a medium access control (MAC) protocol for networked control systems equally challenging. Random access protocols facilitate a distributed implementation, particularly

This work was supported by the Swedish Research Council, VINNOVA (The Swedish Governmental Agency for Innovation Systems), the Swedish Foundation for Strategic Research, the Knut and Alice Wallenberg Foundation and the EU project FeedNetBack.

C. Ramesh, H. Sandberg, L.Bao and K.H.Johansson are with the ACCESS Linnaeus Centre, Electrical Engineering, Royal Institute of Technology (KTH), Stockholm, Sweden. $\{$ cramesh, hsan, lei.bao,kallej\}@ee.kth.se for wireless networks, but they cannot provide a guarantee on performance measures in general. However, by utilizing all the information available to the scheduler, and using this information to determine a probability of channel access, it may be possible to provide performance guarantees with a simple, distributed implementation.

The approaches discussed above motivate the use of a state-based scheduler in the closed loop. Here, we use the term state-based scheduler in a generic sense, to mean that the arrival of a data packet is correlated to the plant state. We are interested in studying the effect of such a scheduler on the control design. Using a quadratic criterion for the cost of controlling a linear plant disturbed by Gaussian noise, we ask, in this paper, if the certainty equivalence principle holds. To answer this question, we examine if there is a dual effect due to the influence of the control action in the arrival of a data packet, as this determines the ease of designing a controller. The lack of a dual effect implies that the certainty equivalence principle holds [3]. Certainty Equivalence is a desirable trait, as the presence of the scheduler can then be ignored and the optimal controller designed with full state information. The lack of state information is compensated with an estimate of the state. However, the presence of a dual effect makes the design of an optimal controller a harder task. In this case, the scheduler cannot be neglected and there is a coupling between the controller, estimator and scheduler.

In this paper, we show that there is a dual effect of the control with state-based scheduling. The optimal controller, scheduler and estimator are coupled and hard to find. We illustrate, with a simple example, some of the computational difficulties in finding the optimal estimates and control signals for a given scheduler. As a suboptimal and simplified approach, we discuss the constraints to be placed on the scheduling criterion such that the resulting system has no dual effect. Consequently, the optimal controller becomes certainty equivalent and for symmetric scheduling criteria, the estimator becomes computationally simple. We note that it is possible to map any state-based scheduler into one which satisfies the constraints required to possess no dual effect. Such transformations or equivalent classes have been constructed, particularly in the problem of encoder design for control over limited data-rate communication channels [4], [5]. In these problems, however, the resulting control actions are not affected by the equivalent design. We show that this is not true for the problem described in this paper. Thus, it is not easy to do away with the dual effect in state-based scheduling and the problem must be solved as such.

The results presented in this paper are built on the classical 
work on dual effect [6] and Certainty Equivalence (CE) [3], [7], as applied to, for instance, adaptive control [8]. In networked control, state-based scheduling has recently been discussed in [9] and [10]. It has not been made sufficiently clear in the recent literature that the CE controller obtained is with a possibly sub-optimal scheduler. In this paper, we highlight some of the difficulties in optimal controller design with a state-based scheduler in the closed loop. The rest of the paper is organized as follows. In section II, we discuss the system architecture and the notation used in the paper. The main results are presented in the form of theorems and propositions in section III. The optimal controller and the $\mathrm{CE}$ controller for an equivalent scheduler are presented in section IV, followed by the conclusion.

\section{PROBLEM SETUP}

We consider a single plant and controller, which communicate over a network with a state-based scheduler in the loop, as shown in Fig. 1. The plant $\mathcal{P}$ has state dynamics given by

$$
x_{k+1}=A x_{k}+B u_{k}+w_{k},
$$

where $A \in \mathbb{R}^{n \times n}, B \in \mathbb{R}^{n \times m}$ and $w_{k}$ is an i.i.d. zero-mean Gaussian noise with covariance matrix $R_{w}$. The initial state $x_{0}$ is a zero-mean Gaussian with covariance matrix $R_{0}$.

There is a scheduler $\mathcal{S}$ between the plant and the controller $\mathcal{C}$, which decides if the state is to be sent to the controller or not. The measurement at the controller is denoted $y_{k}=\delta_{k} x_{k}$, where $\delta_{k} \in\{0,1\}$ is the scheduler output. It takes a value 1 when the state $x_{k}$ is sent and 0 otherwise.

The scheduling criterion $f$ and the control law $g$ denote admissible policies for the finite horizon $N$ defined on the information patterns of the scheduler and the controller, $\mathbb{I}_{k}^{S}$ and $\mathbb{I}_{k}^{C}$, respectively, as given by

$$
\begin{array}{ll}
\delta_{k}=f_{k}\left(\mathbb{I}_{k}^{S}\right) ; & \mathbb{I}_{k}^{S}=\left[\boldsymbol{x}_{0}^{k}, \boldsymbol{y}_{0}^{k-1}, \boldsymbol{\delta}_{0}^{k-1}, \boldsymbol{u}_{0}^{k-1}\right] \\
u_{k}=g_{k}\left(\mathbb{I}_{k}^{C}\right) ; & \mathbb{I}_{k}^{C}=\left[\boldsymbol{y}_{0}^{k}, \boldsymbol{\delta}_{0}^{k}, \boldsymbol{u}_{0}^{k-1}\right]
\end{array}
$$

where the history of a variable is denoted by the boldface notation, as in $\boldsymbol{x}_{0}^{k}=\left\{x_{0}, \ldots, x_{k}\right\}$. The objective function, defined over a horizon $N$ is given by

$$
J(f, g)=\mathbb{E}\left[x_{N}^{T} Q_{0} x_{N}+\sum_{s=0}^{N-1} x_{s}^{T} Q_{1} x_{s}+u_{s}^{T} Q_{2} u_{s}\right]
$$

where $Q_{0}, Q_{1}$ and $Q_{2}$ are positive definite weighting matrices.

\section{A. Dual Effect}

Note that the control $u_{k}$ might affect the future state uncertainty, in addition to its direct effect on the state. This is called the dual effect of control [6].

Definition 1: A control signal is said to have no dual effect of order $r \geq 2$, if

$$
\mathbb{E}\left[M_{k}^{r} \mid \mathbb{I}_{k}^{C}\right]=\mathbb{E}\left[M_{k}^{r} \mid x_{0}, \boldsymbol{w}_{0}^{\tau_{k}}\right]
$$

where $M_{k}^{r}=\mathbb{E}\left[\left(x_{k}-\mathbb{E}\left[x_{k} \mid \mathbb{I}_{k}^{C}\right]\right)^{r} \mid \mathbb{I}_{k}^{C}\right]$ is the $r^{\text {th }}$ central moment of $x_{k}$ conditioned on $\mathbb{I}_{k}^{C}$ and $\tau_{k}$ is the time index of the last received measurement at time $k$ [3].

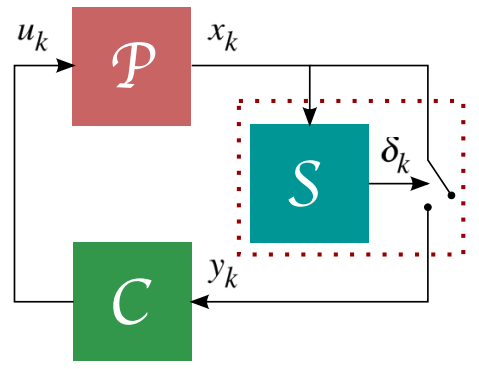

Fig. 1. The system architecture with a state based scheduler.

Note that $M_{k}^{r}$ in (5) must specifically not be a function of the past control policies $\left\{g_{0}, \ldots, g_{k-1}\right\}$ for the control signal to have no dual effect of order $r$. In other words, if there is no dual effect, the expected future uncertainty is not affected by the controls $\boldsymbol{u}_{0}^{k-1}$. In the presence of a dual effect, the optimal control laws are hard to define [8].

\section{B. Certainty Equivalence}

Definition 2: The Certainty Equivalence Principle is said to hold if the closed-loop optimal controller has the same form as the deterministic optimal controller with the state $x_{k}$ replaced by the estimate $\hat{x}_{k \mid k}=\mathbb{E}\left[x_{k} \mid \mathbb{I}_{k}^{C}\right]$.

In contrast, a certainty equivalent controller uses the deterministic optimal controller, with the state $x_{k}$ replaced by the estimate $\hat{x}_{k \mid k}$, as an ad hoc procedure [11], [3].

In the rest of the paper, we address the question of whether the control problem described in this section has a dual effect or not.

\section{MAIN RESULTS}

In this section, we show that the control signal has a dual effect in the presence of a state-based scheduler in the closed loop. We derive the conditions on the scheduling function $f$ for which the system has no dual effect, and discuss how a transformation of the state-based scheduler into one without a dual effect results in another control system, not an equivalent one. Finally, we comment on the structure of the estimator.

\section{A. Dual Effect}

For the problem defined in section II, the following result on the dual effect can be stated.

Theorem 1: The control signal for the plant (1) with statebased scheduler (2), controller (3) and cost criterion (4) has a dual effect of order $r=2$.

Proof: We examine the estimation error covariance $P_{k \mid k}$, and show that it is a function of the applied control signals $\boldsymbol{u}_{0}^{k-1}$. The measurement $y_{k}$ contains the full state $x_{k}$ only when a packet arrives, or when $\delta_{k}=1$. Thus, we have an expression for the estimate $\hat{x}_{k \mid k} \triangleq \mathbb{E}\left[x_{k} \mid \mathbb{I}_{k}^{C}\right]$, as given by

$$
\hat{x}_{k \mid k}= \begin{cases}x_{k} & \delta_{k}=1 \\ \mathbb{E}\left[x_{k} \mid \mathbb{I}_{k}^{C}, \delta_{k}=0\right] & \delta_{k}=0\end{cases}
$$

The estimation error, $\tilde{x}_{k \mid k} \triangleq x_{k}-\hat{x}_{k \mid k}$, can be written as

$$
\tilde{x}_{k \mid k}= \begin{cases}0 & \delta_{k}=1 \\ x_{k}-\mathbb{E}\left[x_{k} \mid \mathbb{I}_{k}^{C}, \delta_{k}=0\right] & \delta_{k}=0\end{cases}
$$


Then, the error covariance, $P_{k \mid k} \triangleq \mathbb{E}\left[\tilde{x}_{k \mid k} \tilde{x}_{k \mid k}^{T} \mid \mathbb{I}_{k}^{C}\right]$, is given by

$$
P_{k \mid k}= \begin{cases}0 & \delta_{k}=1 \\ \mathbb{E}\left[\tilde{x}_{k \mid k} \tilde{x}_{k \mid k}^{T} \mid \mathbb{I}_{k}^{C}, \delta_{k}=0\right] & \delta_{k}=0\end{cases}
$$

The covariance $P_{k \mid k}$ is zero if the scheduling criterion (2) is fulfilled, and non-zero otherwise. Clearly, $P_{k \mid k}$ is a function of the past controls, as the scheduler outcome $\delta_{k}$ is influenced by the applied control inputs $\boldsymbol{u}_{0}^{k-1}$. Since $P_{k \mid k}$ does not satisfy the condition (5) required to have no dual effect, we see that the system (1)-(4) exhibits a dual effect of order $r=2$.

In this setup, the control signal can probe the state, due to which it has influence over the scheduler outcome, and consequently over the estimation error. This provides an incentive to the control policy to modify the estimation error along with controlling the plant. Thus, the optimal controller is not certainty equivalent. This can also be noted from the classical result of Bar-Shalom and Tse [3], which says that the certainty equivalence principle does not hold for a system, such as (1)-(4), which exhibits a dual effect.

Note that the above result implies that the dual effect is visible in any control signal applied to the plant, not just the optimal one, as the control signal will always influence the estimation error, irrespective of whether it has been designed to do so or not. Finally, in this context, the dual effect can be best explained as a coupling between the control and scheduling policies. The information at the scheduler contains all the information available to the controller. Despite this, the control policy can signal to the estimation error as the scheduler outcome is a function of the control design.

\section{B. Conditions for Certainty Equivalence}

A scheduling criterion independent of the past control actions results in no dual effect. This statement is formalized in the theorem below on certainty equivalence.

Theorem 2: The optimal controller for the system (1)-(4) is certainty equivalent if the scheduling decisions are not a function of the applied control actions, i.e. if

$$
\delta_{k}=\tilde{f}_{k}\left(x_{0}, \boldsymbol{w}_{0}^{k-1}\right)
$$

Proof: To minimize the quadratic cost $J$ (4), we need to find a solution to the Bellman equation [11], which is a one-step minimization of the form

$$
V_{k}=\min _{u_{k}} \mathbb{E}\left[x_{k}^{T} Q_{1} x_{k}+u_{k}^{T} Q_{2} u_{k}+V_{k+1} \mid \mathbb{I}_{k}^{C}\right]
$$

In general, without defining a structure for the estimator, BarShalom and Tse [3] give us the solution to the functional, of the form

$$
V_{k}=\mathbb{E}\left[x_{k}^{T} S_{k} x_{k} \mid \mathbb{I}_{k}^{C}\right]+s_{k}
$$

where $S_{k}$ is a positive semi-definite matrix and both $S_{k}$ and $s_{k}$ are not functions of the applied control signals $\boldsymbol{u}_{0}^{k-1}$. We now prove that a solution of this form can be found for our problem with the scheduler (7).

At time $N$, the functional has a trivial solution (9) with $S_{N}=Q_{0}$ and $s_{N}=0$. This solution can be propagated backwards, in the absence of a dual effect. To show this, we use the principle of induction, and assume the solution to hold at time $k+1$. Then, at time $k$, we have

$$
\begin{array}{rl}
V_{k}=\min _{u_{k}} & \mathbb{E}\left[x_{k}^{T} Q_{1} x_{k}+u_{k}^{T} Q_{2} u_{k}+x_{k+1}^{T} S_{k+1} x_{k+1}+s_{k+1} \mid \mathbb{I}_{k}^{C}\right] \\
=\min _{u_{k}} & \mathbb{E}\left[x_{k}^{T}\left(Q_{1}+A^{T} S_{k+1} A\right) x_{k} \mid \mathbb{I}_{k}^{C}\right]+\operatorname{tr}\left\{S_{k+1} R_{w}\right\} \\
& +\mathbb{E}\left[s_{k+1} \mid \mathbb{I}_{k}^{C}\right]+u_{k}^{T}\left(Q_{2}+B^{T} S_{k+1} B\right) u_{k} \\
& +\hat{x}_{k \mid k}^{T} A^{T} S_{k+1} B u_{k}+u_{k}^{T} B^{T} S_{k+1} A \hat{x}_{k \mid k}
\end{array}
$$

The optimal control is then found to be

$$
u_{k}=-\left(Q_{2}+B^{T} S_{k+1} B\right)^{-1} B^{T} S_{k+1} A \hat{x}_{k \mid k}
$$

Substituting the expression for $u_{k}$ into $V_{k}$ gives us a solution of the form in (9), with

$$
\begin{aligned}
S_{k}= & Q_{1}+A^{T} S_{k+1} A \\
& -A^{T} S_{k+1} B\left(Q_{2}+B^{T} S_{k+1} B\right)^{-1} B^{T} S_{k+1} A \\
s_{k}= & \operatorname{tr}\left\{S_{k+1} R_{w}\right\}+\mathbb{E}\left[s_{k+1} \mid \mathbb{I}_{k}^{C}\right] \\
& +\operatorname{tr}\left\{A^{T} S_{k+1} B\left(Q_{2}+B^{T} S_{k+1} B\right)^{-1} B^{T} S_{k+1} A P_{k \mid k}\right\}
\end{aligned}
$$

where the matrix $S_{k}$ is positive semi-definite and not a function of the applied controls $\boldsymbol{u}_{0}^{k-1}$. The scalar $s_{k}$ is not a function of the applied controls $\boldsymbol{u}_{0}^{k-1}$ if and only if $P_{k \mid k}$ has no dual effect [3]. From the expression for the error covariance $P_{k \mid k}$ (6), it is clear that a scheduling criterion that is not a function of the past control actions, such as (7), results in no dual effect. Under this condition, $s_{k}$ is not a function of the applied controls $\boldsymbol{u}_{0}^{k-1}$ and the proof by induction is complete. Since the optimal control signal (10) is a function of only the estimate $\hat{x}_{k \mid k}$, Certainty Equivalence holds.

Theorem 2 gives a condition on the scheduler to guarantee certainty equivalence. Note that the resulting closed loop system is not optimal in general. A scheduler with a dual effect may result in a better design with lower cost.

\section{Equivalent Classes for the Scheduler}

We now examine the question of whether this dual effect can be removed by the construction of an equivalent scheduler. Following the results presented in subsections III-A and III-B, it is tempting to note that every state-based scheduler $f(2)$ can be transformed into an equivalent scheduler $\tilde{f}$ (7), as the applied controls $\boldsymbol{u}_{0}^{k-1}$ are known at time $k$ [4]. This theoretical construct implies that $f$ and $\tilde{f}$ result in the same set of transmission instants for a given system. In particular, if the optimal control policy for the system with scheduling criterion $f$ is denoted $g^{*}$, and the optimal certainty equivalent control policy for the system with scheduling criterion $\tilde{f}$ is denoted $g_{\mathrm{CE}}$, we show that the system $\left\{\tilde{f}, g_{\mathrm{CE}}\right\}$ is not an equivalent replacement for the system with $\left\{f, g^{*}\right\}$. We begin with a definition of an equivalent system.

Equivalent System: In [7], Witsenhausen notes that two designs $g$ and $g$ att are equivalent when they result in the same control signals for a given set of primitive random variables $\boldsymbol{\omega}$, as this implies that the joint distribution of all system variables is the same with either design. 
In the context of our problem, we define equivalent systems, in the sense of Witsenhausen, as

Definition 3: An equivalent control design $g_{\mathrm{eq}}$ for the optimal controller $g^{*}$, which minimizes the cost criterion (4) for the system defined by (1)-(3), satisfies the equivalence relationship given by $\boldsymbol{u}^{*}=S\left(\boldsymbol{\omega}, g^{*}\right)=S_{\mathrm{eq}}\left(\boldsymbol{\omega}, g_{\mathrm{eq}}\right)$, where the relations $S$ and $S_{\text {eq }}$ can be obtained by recursive substitution for the control policies $g^{*}$ and $g_{\text {eq }}$, respectively.

Due to the dual effect, the optimal control action, given by the design $g^{*}$, takes on two roles. One, to control the plant, and the other, to probe the plant state which could result in an improved estimate [8],[12] and [13]. In the certainty equivalent setup, the probing action cannot be implemented due to the lack of a dual effect. The resulting control actions will not remain the same, i.e. $g_{\mathrm{CE}}$ does not belong to the class of equivalent controllers such as $g_{\text {eq }}$. Let $\{\mathcal{P}, \bar{f}, \bar{g}\}$ denote the system (1)-(3), with $\bar{f}$ as the given scheduler and $\bar{g}$ as the optimal controller for the cost criterion (4) applied to this system. We now observe the following result.

Theorem 3: For two schedulers $f$, given by (2) and $\tilde{f}$, given by (7), which result in the same schedules for the system (1)-(3), $\left\{\mathcal{P}, \tilde{f}, g_{\mathrm{CE}}\right\}$ is not an equivalent system to $\left\{\mathcal{P}, f, g^{*}\right\}$.

Proof: Definition 3 requires the resulting control signals from $g^{*}$ and $g_{\mathrm{CE}}$ to be equal. We show that this is not possible. Solving the backward recursion as we did in the proof of theorem 2, we find that $V_{N}$ and $V_{N-1}$ for both systems have a solution of the form (9), with $S_{N}=Q_{0}$ and $s_{N}=0$, and $S_{N-1}$ and $s_{N-1}$ given by (11) with $k=N-1$. However, at time $N-2$, the cost-to-go $V_{N-2}$ results in different minimization problems for the two systems because of the dual effect in $\left\{\mathcal{P}, f, g^{*}\right\}$.

For the system $\left\{\mathcal{P}, f, g_{\mathrm{CE}}\right\}$, due to its certainty equivalent property, the optimal control signal $u_{N-2}^{\mathrm{CE}}$ is obtained by minimizing $V_{N-2}$ over $u_{N-2}^{\mathrm{CE}}$, to get

$$
\begin{aligned}
\frac{\partial V_{N-2}}{\partial u_{N-2}^{\mathrm{CE}}}= & 2 u_{N-2}^{\mathrm{CE} T}\left(Q_{2}+B^{T} S_{N-1} B\right) \\
& +2 \hat{x}_{N-2 \mid N-2}^{T} A^{T} S_{N-1} B=0
\end{aligned}
$$

However, the system $\left\{\mathcal{P}, f, g^{*}\right\}$ is not certainty equivalent, and the optimal control signal $u_{N-2}^{*}$ can be obtained by solving

$$
\begin{aligned}
\frac{\partial V_{N-2}}{\partial u_{N-2}^{*}}= & 2 u_{N-2}^{* T}\left(Q_{2}+B^{T} S_{N-1} B\right)+2 \hat{x}_{N-2 \mid N-2}^{T} A^{T} S_{N-1} B \\
& +\frac{\partial}{\partial u_{N-2}^{*}}\left(\operatorname { t r } \left\{A^{T} S_{N} B\left(Q_{2}+B^{T} S_{N} B\right)^{-1} B^{T} S_{N} A\right.\right. \\
& \left.\left.\cdot \mathbb{E}\left[P_{N-1 \mid N-1} \mid \mathbb{I}_{N-2}^{C}\right]\right\}\right)=0
\end{aligned}
$$

This is obtained by substituting the solution to the function $V_{N-1}$ into the minimization problem at $N-2$. From Theorem 1, we know that the additional term in (13) is not zero. Due to this term, the solutions $u_{N-2}^{\mathrm{CE}}$ and $u_{N-2}^{*}$ need not be equal. From this point on, the cost-to-go for the optimal control policy $g^{*}$ does not have a solution of the form given by (9). Hence, the control signals $\left\{\boldsymbol{u}^{\mathrm{CE}}\right\}_{0}^{N-3}$ and $\left\{\boldsymbol{u}^{*}\right\}_{0}^{N-3}$ are not equal and the joint distribution of all system variables can be quite different for schedulers $f$ and $\tilde{f}$. Thus, the described transformation of the scheduling criterion does not result in an equivalent class construction.

\section{Estimator}

In this section, we provide additional constraints on the scheduler that simplify the estimator considerably. Note that the estimation error is reset to zero with every successful transmission. Consider one such reset instant, a time $k$ such that $\delta_{k}=1$. The state is sent across the network, $y_{k}=x_{k}$, so the estimate $\hat{x}_{k \mid k}=x_{k}$. A suitable control signal $u_{k}$ is found and applied to the plant, which results in the next state $x_{k+1}$. Now, the scheduler can generate one of two outcomes. We consider each in detail below:

a) $\delta_{k+1}=0$ : We need an estimate of $w_{k}$. We use the scheduler output as a coarse quantized measurement to generate this, as follows:

$$
\begin{aligned}
\hat{x}_{k+1 \mid k+1} & =\mathbb{E}\left[x_{k+1} \mid \mathbb{I}_{\mathrm{k}+1}^{\mathbb{C}}, \delta_{k+1}=0\right] \\
& =A x_{k}+B u_{k}+\mathbb{E}\left[w_{k} \mid \grave{f}\left(w_{k}\right)=0\right] \\
\tilde{x}_{k+1 \mid k+1} & \triangleq x_{k+1}-\hat{x}_{k+1 \mid k+1}=w_{k}-\mathbb{E}\left[w_{k} \mid \grave{f}\left(w_{k}\right)=0\right]
\end{aligned}
$$

where, $\grave{f}\left(w_{k}\right) \equiv f\left(A x_{k}+B u_{k}+w_{k} \mid x_{k}, u_{k}\right)$.

b) $\delta_{k+1}=1$ : There is no estimation error as $\hat{x}_{k+1 \mid k+1}=$ $x_{k+1}$ and the estimator is reset.

We use the transformation to $\grave{f}(14)$, as it does not affect the control law outcome $\boldsymbol{u}_{0}^{k}$. This transformation is not intended to remove any dual effect, as the dual effect has resulted in the packet being sent or not, i.e., in the value of $\delta_{k+1}$. Once $\delta_{k+1}$ is known, the transformation merely serves to remove the known variables from the expression.

To see this more clearly, we look at the next time instant. So, now a signal $u_{k+1}$ is generated, and applied to the plant. We note that $x_{k+2}=A^{2} x_{k}+A B u_{k}+B u_{k+1}+A w_{k}+$ $w_{k+1}$. The state $x_{k+2}$ is either sent to the controller or not depending on the scheduler outcome $\delta_{k+2}$. Again, we look at both cases:

i) $\delta_{k+2}=0$ : We now need to estimate $A w_{k}+w_{k+1}$, as the rest is completely known from $x_{k+2}$. We use both scheduler outputs $\delta_{k+1}$ and $\delta_{k+2}$ to generate an estimate of the unknown variables as

$$
\begin{aligned}
\hat{x}_{k+2 \mid k+2} & =A^{2} x_{k}+A B u_{k}+B u_{k+1} \\
& +\mathbb{E}\left[A w_{k}+w_{k+1} \mid \grave{f}\left(w_{k}\right)=0, \grave{f}\left(A w_{k}+w_{k+1}\right)=0\right] \\
\tilde{x}_{k+2 \mid k+2} & =A w_{k}+w_{k+1} \\
& -\mathbb{E}\left[A w_{k}+w_{k+1} \mid \grave{f}\left(w_{k}\right)=0, \grave{f}\left(A w_{k}+w_{k+1}\right)=0\right]
\end{aligned}
$$

ii) $\delta_{k+2}=1$ : There is no estimation error as $\hat{x}_{k+2 \mid k+2}=$ $x_{k+2}$ and the estimator is reset.

This process can be continued recursively through a nontransmission burst, until finally a measurement is received and the estimation error is reset to zero. Thus, the estimate at any time $k$ is given by

$$
\hat{x}_{k \mid k}= \begin{cases}x_{k} & \delta_{k}=1 \\ A^{k-\tau_{k}} x_{\tau_{k}}+\sum_{s=1}^{k-\tau_{k}} A^{s-1} B u_{k-s} & \delta_{k}=0 \\ +\mathbb{E}\left[\sum_{s=1}^{k-\tau_{k}} A^{s-1} w_{k-s} \mid \grave{f}_{k}, . ., \grave{f}_{\tau_{k}+1}=0\right] & \end{cases}
$$


where $\tau_{k}$ is the time index of the last received measurement at time $k$ and $\sum_{s=1}^{t-\tau_{t}} A^{s-1} w_{t-s}$ are the arguments of $\grave{f}_{t}$. Note that the computation of the term $\mathbb{E}\left[\sum_{s=1}^{k-\tau_{k}} A^{s-1} w_{k-s} \mid \grave{f}_{k}, . ., \grave{f}_{\tau_{k}+1}=0\right]$ is non-trivial for a burst of non-transmissions of length greater than one, as the quantized noise is not Gaussian. As a sub-optimal, but simplified approach, the scheduling criterion at any time $k$ can be chosen as a symmetric function of the argument $\sum_{s=1}^{k-\tau_{k}} A^{s-1} w_{k-s}$ to obtain a zero mean term from the quantized noise when there is no transmission. Then, the estimate is easy to compute and a certainty equivalent control can be applied, as observed in the following proposition.

Proposition 4: For the system (1)-(4), choosing the scheduling criterion to be a symmetric function of the argument $\sum_{s=1}^{k-\tau_{k}} A^{s-1} w_{k-s}$ results in a computationally simple estimator and an optimal CE controller.

\section{A 2-STEP HORIZON EXAMPLE}

We now look at a simple example to see the computational difficulties in identifying optimal estimates and controls for a system with a state-based scheduler in the closed loop. We also show that for an equivalent scheduler such as $\tilde{f}$ in Section III-C, which is free of a dual effect, the entire plant is altered, so the equivalence construction does not work.

For this example, we consider a scalar plant, given by $x_{k+1}=a x_{k}+b u_{k}+w_{k}$, with $a, b \in \mathbb{R}$ and $x_{0}, w_{k} \sim \mathcal{N}(0,1)$. The packet is scheduled for transmission when $x_{k} \geq 0.5$. The controllers are designed to minimize the LQG cost (4), for a horizon of two steps, i.e., $N=2$, and with $Q_{0}, Q_{1}, Q_{2} \in \mathbb{R}$. In the first subsection, we derive the optimal controller for the scheduler with dual effect. Then, for the same schedule, in the second subsection, we define the certainty equivalent controller, assuming that an equivalent scheduler has been designed without a dual effect. We do not discuss the design of the equivalent scheduler as it is a theoretical construct, which sometimes permits a simplification of the problem. We finally compare the resulting control actions and show that they are not the same, and thus, the equivalent scheduler does not simplify the controller design in our problem.

\section{A. Optimal Controller}

We first derive the estimation error covariances $P_{0 \mid 0}$ and $P_{1 \mid 1}$, as these are used in the derivation of the optimal control signals $u_{0}$ and $u_{1}$. From (15), we get

$$
\hat{x}_{0 \mid 0}= \begin{cases}x_{0} & \delta_{0}=1 \\ \mathbb{E}\left[x_{0} \mid x_{0}<0.5\right] & \delta_{0}=0\end{cases}
$$

As $x_{0} \sim \mathcal{N}(0,1)$, we can find the expected value

$$
\bar{x}_{\delta 0}:=\mathbb{E}\left[x_{0} \mid x_{0}<0.5\right]=\int_{-\infty}^{0.5} x \phi_{x_{\delta 0}}(x) d x
$$

where $\phi_{x_{\delta 0}}$ is the conditional probability distribution function (pdf) of $x_{0}$, conditioned on $x_{0}<0.5$. Thus, $\phi_{x_{\delta 0}}(x)=$ $\phi_{x_{0}}(x) / \operatorname{Pr}\left(x_{0}<0.5\right)$, where $\phi_{x_{0}}$ is the pdf of $x_{0}$. The probability of a non-transmission is given by

$$
\operatorname{Pr}\left(x_{0}<0.5\right)=\int_{-\infty}^{0.5} \phi_{x_{0}}(x) d x
$$

The estimation error $\tilde{x}_{0 \mid 0}$ is non-zero only when $\delta_{0}=0$, and is given by $x_{0}-\bar{x}_{\delta 0}$. The corresponding estimation error covariance $P_{0 \mid 0}=R_{\tilde{x}_{0}}$, which is given by

$R_{\tilde{x}_{0}}=\mathbb{E}\left[\left(x_{0}-\bar{x}_{\delta 0}\right)^{2} \mid x_{0}<0.5\right]=\int_{-\infty}^{0.5-\bar{x}_{\delta 0}} x^{2} \phi_{x_{\delta 0}}\left(x+\bar{x}_{\delta 0}\right) d x$

where, the pdf of $\tilde{x}_{0 \mid 0}$ is $\phi_{\tilde{x}_{0}}(x)=\phi_{x_{\delta 0}}\left(x+\bar{x}_{\delta 0}\right)$.

Let $e_{1}$ denote the unknown part of $x_{1}$ before $y_{1}$ is received:

$$
e_{1}=\left\{\begin{array}{ll}
w_{0} & \delta_{0}=1 \\
a x_{0}+w_{0} & \delta_{0}=0
\end{array} \quad \phi_{e}(\epsilon)= \begin{cases}\phi_{w_{0}}(\epsilon) & \delta_{0}=1 \\
\phi_{e_{\delta 0}}(\epsilon) & \delta_{0}=0\end{cases}\right.
$$

where $\phi_{e}$ is the pdf of $e_{1}$. The variable $e_{1}$ is the sum of two random variables if $\delta_{0}=0$, and its pdf is denoted $\phi_{e_{\delta 0}}$, and given by

$$
\begin{aligned}
\phi_{e_{\delta 0}}(\epsilon) & =\int_{-\infty}^{0.5} \phi_{x_{\delta 0}}(x) \phi_{w_{0}}(\epsilon-a x) d x \\
& =\frac{e^{-\epsilon^{2} / 2\left(1+a^{2}\right)}}{\sqrt{2 \pi\left(1+a^{2}\right)}}\left[\frac{\operatorname{Pr}\left(t<\frac{1+a^{2}-2 a \epsilon}{2 \sqrt{1+a^{2}}}\right)}{\operatorname{Pr}\left(x_{0}<0.5\right)}\right]
\end{aligned}
$$

where, $t \sim \mathcal{N}(0,1)$. Then, at the next time instant, we get

$$
\hat{x}_{1 \mid 1}=\left\{\begin{array}{lll}
x_{1} & \delta_{1}=1 \\
\begin{cases}a x_{0}+b u_{0}+\bar{w}_{0} & \delta_{0}=1 \\
b u_{0}+\bar{e}_{\delta 0} & \delta_{0}=0\end{cases} & \delta_{1}=0
\end{array}\right.
$$

As $w_{0} \sim \mathcal{N}(0,1)$, we can find the expected value

$\bar{w}_{0}=\mathbb{E}\left[w_{0} \mid w_{0}<0.5-a x_{0}-b u_{0}\right]=\int_{-\infty}^{0.5-a x_{0}-b u_{0}} w \phi_{w_{0}}(w) d w$

where $\phi_{w_{0}}$ is the pdf of $w_{0}$. Similarly, using the expression for $\phi_{e_{\delta 0}}$, we can derive

$$
\begin{aligned}
\bar{e}_{\delta 0} & =\mathbb{E}\left[a x_{0}+w_{0} \mid x_{0}<0.5, a x_{0}+w_{0}<0.5-b u_{0}\right] \\
& =\frac{1}{\operatorname{Pr}\left(e_{1}<0.5-b u_{0}\right)} \int_{-\infty}^{0.5-b u_{0}} \epsilon \phi_{e_{\delta 0}}(\epsilon) d \epsilon
\end{aligned}
$$

where $\operatorname{Pr}\left(e_{1}<0.5-b u_{0}\right)$ is the probability of no transmission at time $k=1$. We know that

$$
\operatorname{Pr}\left(e_{1}<0.5-b u_{0}\right)=\int_{-\infty}^{0.5-b u_{0}} \phi_{e_{\delta 0}}(\epsilon) d \epsilon
$$

We now define $\tilde{e}_{1}$ as the error in estimating the term $e_{1}$ after $y_{1}$ arrives, with pdf $\phi_{\tilde{e}}$, so that

$$
\begin{aligned}
\tilde{e}_{1} & = \begin{cases}w_{0}-\bar{w}_{0} & \delta_{0}=1 \\
a x_{0}+w_{0}-\bar{e}_{\delta 0} & \delta_{0}=0\end{cases} \\
\phi_{\tilde{e}}(\epsilon) & = \begin{cases}\phi_{\tilde{w}}\left(\epsilon+\bar{w}_{0} \mid w_{0}<0.5-a x_{0}-b u_{0}\right) & \delta_{0}=1 \\
\phi_{e_{\delta 0}}\left(\epsilon+\bar{e}_{\delta 0} \mid e_{1}<0.5-b u_{0}\right) & \delta_{0}=0\end{cases}
\end{aligned}
$$

Now, the estimation error variance $P_{1 \mid 1}=R_{e_{1}}$ when $\delta_{1}=$ 0 , and zero otherwise. Here, $R_{e_{1}}=\mathbb{E}\left[\tilde{e}_{1}^{2} \mid \delta_{1}=0\right]$ is given by

$R_{e_{1}}= \begin{cases}\int_{-\infty}^{0.5-a x_{0}-b u_{0}-\bar{w}_{0}} w^{2} \frac{\phi_{w_{0}}\left(w+\bar{w}_{0}\right)}{\operatorname{Pr}\left(w_{0}<0.5-a x_{0}-b u_{0}\right)} d w & \delta_{0}=1 \\ \int_{-\infty}^{0.5-b u_{0}-\bar{e}_{\delta 0}} \epsilon^{2} \frac{\phi_{\delta 0}\left(\epsilon+\bar{e}_{\delta 0}\right)}{\operatorname{Pr}\left(e_{1}<0.5-b u_{0}\right)} d \epsilon & \delta_{0}=0\end{cases}$ 
To solve for the optimal control signals, we use $V_{1}$ and $V_{0}$ from (8). Using the principles of dynamic programming, we find the $u_{1}$ that minimizes $V_{1}$ to be

$$
u_{1}=-\frac{a b Q_{0}}{Q_{2}+b^{2} Q_{0}} \hat{x}_{1 \mid 1}
$$

Substituting for $u_{1}$ in the above expression for $V_{1}$, we get

$$
V_{1}=\mathbb{E}\left[x_{1}^{2} S_{1}+\operatorname{tr}\left\{\frac{a^{2} Q_{0}^{2} b^{2}}{Q_{2}+b^{2} Q_{0}} P_{1 \mid 1}\right\} \mid \mathbb{I}_{1}^{\mathbb{C}}\right]+\operatorname{tr}\left\{Q_{0} R_{w}\right\}
$$

where $S_{1}=Q_{1}+a^{2} Q_{0}-\frac{a^{2} Q_{0}^{2} b^{2}}{Q_{2}+b^{2} Q_{0}}$. To derive $V_{0}$, we need to find the expected value $\mathbb{E}\left[P_{1 \mid 1} \mid \mathbb{I}_{0}^{\mathbb{C}}\right]$. From the definition of $P_{1 \mid 1}$, we find that $\mathbb{E}\left[P_{1 \mid 1} \mid \mathbb{I}_{0}^{\mathbb{C}}\right]=\operatorname{Pr}\left(\delta_{1}=0 \mid \mathbb{I}_{0}^{\mathbb{C}}\right) \mathbb{E}\left[R_{e_{1}} \mid \mathbb{I}_{0}^{\mathbb{C}}\right]$. Then, we can find $u_{0}$ that minimizes $V_{0}$, by solving

$$
\begin{aligned}
\frac{\partial V_{0}}{\partial u_{0}}= & 2 u_{0}\left(Q_{2}+b^{2} S_{1}\right)+2 \hat{x}_{0 \mid 0} a b S_{1} \\
& +\frac{a^{2} Q_{0}^{2} b^{2}}{Q_{2}+b^{2} Q_{0}} \cdot \frac{\partial}{\partial u_{0}}\left(\mathbb{E}\left[P_{1 \mid 1} \mid \mathbb{I}_{0}^{\mathbb{C}}\right]\right)=0
\end{aligned}
$$

This can be simplified using the expression for $R_{e_{1}}$, to get $\frac{\partial V_{0}}{\partial u_{0}}=2 u_{0}\left(Q_{2}+b^{2} S_{1}\right)+2 \hat{x}_{0 \mid 0} a b S_{1}-\frac{a^{2} Q_{0}^{2} b^{2}}{Q_{2}+b^{2} Q_{0}} K=0$, where $K=b\left(w_{0, \max }-\bar{w}_{0}\right)^{2} \phi_{w_{0}}\left(w_{0, \max }\right)$ when $\delta_{0}=1$ and $K=b\left(e_{\max }-\bar{e}_{\delta 0}\right)^{2} \phi_{e_{\delta 0}}\left(e_{\max }\right)$ when $\delta_{0}=0$. In these expressions, $w_{0, \max }=0.5-a x_{0}-b u_{0}$ and $e_{\max }=0.5-b u_{0}$. The final equation is obtained using Leibnitz rule. Solving these equations give the optimal $u_{0}$.

\section{B. CE Controller}

For the same scheduler outcomes $\delta_{0}, \delta_{1}$ obtained through an equivalent scheduler which has no dual effect, the certainty equivalent controller gives us the control signals

$$
u_{1}=-\frac{A b Q_{0}}{Q_{2}+b^{2} Q_{0}} \hat{x}_{1 \mid 1}, u_{0}=-\frac{A b S_{1}}{Q_{2}+b^{2} S_{1}} \hat{x}_{0 \mid 0}
$$

Note that $u_{1}$ is found by minimizing $V_{1}$, which results in the same expression as for the optimal controller (16). However, $u_{0}$ for the $\mathrm{CE}$ controller is obtained by solving the equation

$$
2 u_{0}\left(Q_{2}+b^{2} S_{1}\right)+2 \hat{x}_{0 \mid 0} a b S_{1}=0
$$

\section{Discussion}

A comparison of the control signals for the CE controller (18) with $u_{1}$ and $u_{0}$ obtained in (16) and (17), shows that the signal $u_{1}$ remains the same. However, $u_{0}$ is different, and displays a dual effect in the optimal controller. From (19), it is clear that the additional term in (17) alters the solution for the optimal controller. This observation can be explained as follows. In a controller with a dual effect, the control signal can be chosen to probe the plant state in order to improve the quality of the estimate. However, there is no motive in improving the estimate in a one-step optimization process. Thus, $u_{1}$ is the same for both controllers. When the optimization is performed over two steps, a probing effect in the first step can improve the estimate and the corresponding control applied in the next step. Thus, $u_{0}$ is different in the optimal controller for a state-based scheduler.

This example shows us that even the same schedule can result in a different control sequence for a system without a dual effect. Thus, an equivalent construction for the scheduler does not result in an equivalent system in our setup.

\section{CONCLUSION}

We examined the design of an optimal controller with a state-based scheduler in the closed loop. The state dependent scheduler permits a probe of the state by the control signal to effect an improvement in the future estimation error, and thus the system exhibits a dual effect. This dual effect does not exist in a system where the arguments of the scheduling criterion are independent of the past control actions, but this system is not equivalent to the original system even if the resulting schedules are similar. Thus, certainty equivalence can be achieved at the cost of optimality in this problem. We also discussed a structure for the scheduler, and constraints on the scheduling criterion which simplified computations for this problem.

\section{ACKNOWLEDGMENTS}

The authors are grateful for the discussions with Maben Rabi, whose insights and comments have helped to shape this work.

\section{REFERENCES}

[1] K. J. Åström and B. Bernhardsson, "Comparison of Riemann and Lebesgue sampling for first order stochastic systems," Proceedings of the IEEE Conference on Decision and Control (CDC'02), vol. 2, pp. 2011-2016, December 2002.

[2] Y. Xu and J. P. Hespanha, "Optimal communication logics in networked control systems," 43rd IEEE Conference on Decision and Control (CDC '04), vol. 4, pp. 3527-3532, December 2004.

[3] Y. Bar-Shalom and E. Tse, "Dual effect, certainty equivalence, and separation in stochastic control," IEEE Transactions on Automatic Control, vol. 19, pp. 494-500, October 1974.

[4] S. Tatikonda, A. Sahai, and S. Mitter, "Stochastic linear control over a communication channel," IEEE Transactions on Automatic Control, vol. 49, pp. 1549-1561, September 2004.

[5] G. N. Nair, F. Fagnani, S. Zampieri, and R. J. Evans, "Feedback control under data rate constraints: An overview," Proceedings of IEEE special issue on Technology of Networked Control Systems, vol. 95, pp. 108137, 2007.

[6] A. A. Feldbaum, "The theory of dual control. IV," Automat. Remote Control, vol. 22, pp. 109-121, 1961.

[7] H. S. Witsenhausen, "Separation of estimation and control for discrete time systems," Proceedings of the IEEE, vol. 59, pp. 1557-1566, November 1971.

[8] K. J. Åström and B. Wittenmark, Adaptive Control. Addison-Wesley, 1995.

[9] A. Molin and S. Hirche, "On LQG joint optimal scheduling and control under communication constraints," Preprints of the Proc. 48th IEEE Conference on Decision and Control, pp. 5832-5838, December 2009.

[10] C. Ramesh, H. Sandberg, and K. H. Johansson, "LQG and medium access control," Preprints of the 1st IFAC Workshop on Estimation and Control of Networked Systems (NecSys2009), pp. 328-333, September 2009.

[11] K. J. Åström, Introduction to Stochastic Control Theory. Dover Publications, 2006.

[12] N. Filatov and H. Unbehauen, "Survey of adaptive dual control methods," IEEE Proceedings Control Theory Appl., vol. 147, pp. 118$128,2000$.

[13] B. Wittenmark, "Adaptive dual control," tech. rep., http://www.control.lth.se/ bjorn /seminal/dual.pdf. 